\title{
Intracerebral Hemorrhage, Diffusion Restriction and Contrast Enhancement on Magnetic Resonance Imaging in Posterior Reversible Encephalopathy Syndrome Secondary to Antepartum Eclampsia: Case Report and Review of Literature
}

Saurabh Bansal ${ }^{1}$, Ramandeep Bansal ${ }^{2}$, Manoj Kumar Goyal ${ }^{1}$, Paramjeet Singh ${ }^{3}$ and Vivek Lal ${ }^{1 *}$

${ }^{1}$ Department of neurology, Postgraduate Institute of Medical Education and Research, Chandigarh, India

${ }^{2}$ Department of Obstetrics and Gynecology, Postgraduate Institute of Medical Education and Research, Chandigarh, India

${ }^{3}$ Department of Radiology, Postgraduate Institute of Medical Education and Research, Chandigarh, India

Correspondence to:

Vivek Lal, Professor and Head

Department of Neurology, Postgraduate Institute of Medical Education and Research

Chandigarh, India

Tel: 9872155572

E-mail: vivek144@yahoo.com

Received: August 30, 2018

Accepted: November 19, 2018

Published: November 22, 2018

Citation: Bansal S, Bansal R, Goyal MK, Singh P, Lal V. 2018. Intracerebral Hemorrhage, Diffusion Restriction and Contrast Enhancement on Magnetic Resonance Imaging in Posterior Reversible Encephalopathy Syndrome Secondary to Antepartum Eclampsia: Case Report and Review of Literature $J$ Neuroimaging Psychiatry Neurol 3(2): 27-29.

Copyright: (C) 2018 Bansal et al. This is an Open Access article distributed under the terms of the Creative Commons Attribution 4.0 International License (CC-BY) (http://creativecommons. org/licenses/by/4.0/) which permits commercial use, including reproduction, adaptation, and distribution of the article provided the original author and source are credited.

Published by United Scientific Group

\begin{abstract}
Background: The diagnosis of posterior reversible encephalopathy syndrome (PRES) is based on a characteristic radiological picture in the appropriate clinical setting. Knowledge of atypical radiological presentations of PRES is essential to avoid misdiagnosis.
\end{abstract}

Methods: We present a PRES patient secondary to antepartum eclampsia with atypical radiological manifestations and briefly review the literature regarding radiology of PRES.

Results: A 23-year-old woman presented with headache, vomiting, visual disturbances, seizures and encephalopathy at 30 weeks gestational age with blood pressure of 146/100 $\mathrm{mm} \mathrm{Hg}$. She was diagnosed with eclampsia and PRES based on clinical signs, magnetic resonance imaging (MRI) and computed tomographic scanning of the brain revealing signal changes suggestive of PRES. In addition to changes typical of PRES, there was evidence of cerebellar involvement, diffusion restriction and bleeding in the right temporo-parietal regions, changes which are considered atypical for PRES. She was managed conservatively and improved gradually. Repeat MRI done after three months showed resolution of signal abnormalities. At eight months follow up, she has had a complete recovery.

Conclusions: Knowledge of atypical radiological manifestations of PRES helps clinicians in identifying this rare, potentially reversible disorder and avoid unnecessary diagnostic and therapeutic interventions. Our case further aids to the repertoire of atypical radiological findings of PRES.

\section{Keywords}

PRES, Eclampsia, Visual disturbances

\section{Introduction}

Diagnosis of posterior reversible encephalopathy syndrome (PRES) is based on clinical and radiological characteristics. The classic magnetic resonance imaging (MRI) findings described in PRES include bilateral signal changes [hyperintensities on T2 and Fluid attenuation inversion recovery (FLAIR) sequences and hypointense signal changes on T1 weighted MR sequences] in parieto-occipital regions [1, 2]. With increasing use of MRI in neurological disorders, atypical radiological patterns are being encountered in PRES, knowledge of which is essential to avoid diagnostic and therapeutic errors. Here, we present a patient with presumed PRES with unusual imaging features and briefly review the literature. 


\section{Case Report}

A 23-year-old woman presented to our hospital with a one-day history of headache, vomiting, visual disturbances, seizures and encephalopathy at 30 weeks of gestation. Her blood pressure was 146/100 $\mathrm{mm} \mathrm{Hg}$ with severe intrauterine growth retardation on ultrasonography (estimated fetal weight of 568 grams). On neurological examination she was dull and drowsy. There were no meningeal signs. Detailed neurological examination including fundus evaluation was normal except for extensor plantar response. Laboratory investigations were normal save for mild anemia (Hemoglobin: $9 \mathrm{mg} / \mathrm{dL}$ ). A non-contrast enhanced computed tomography (NCCT) scan of head was consistent with a right temporo-parietal bleed with bilateral parieto-occipital and symmetrical cerebellar hypodensities (Figure 1a and 1b). On the basis of clinical and radiological findings a diagnosis of antepartum eclampsia with hemorrhagic PRES was made. Other possibilities considered were cerebral venous sinus thrombosis and reversible cerebral vasospasm which can mimic PRES on computed tomography. She was managed with termination of pregnancy, intravenous magnesium sulphate and supportive care. A gadoliniumenhanced MRI scan of the brain (employing T1 and T2 weighted MR sequences, FLAIR sequences, Diffusion weighted imaging (DWI) sequences with apparent diffusion coefficient maps, susceptibility weight imaging sequences, magnetic resonance angiography and contrast enhanced $\mathrm{T} 1$ weighted images) done four days later on a 1.5 Telsa MR machine revealed signal changes bilaterally in the parietooccipito- temporal regions (right $>$ left) (Figure 1c and 1d) with evidence of hemorrhage (Figure 1c) and diffusion

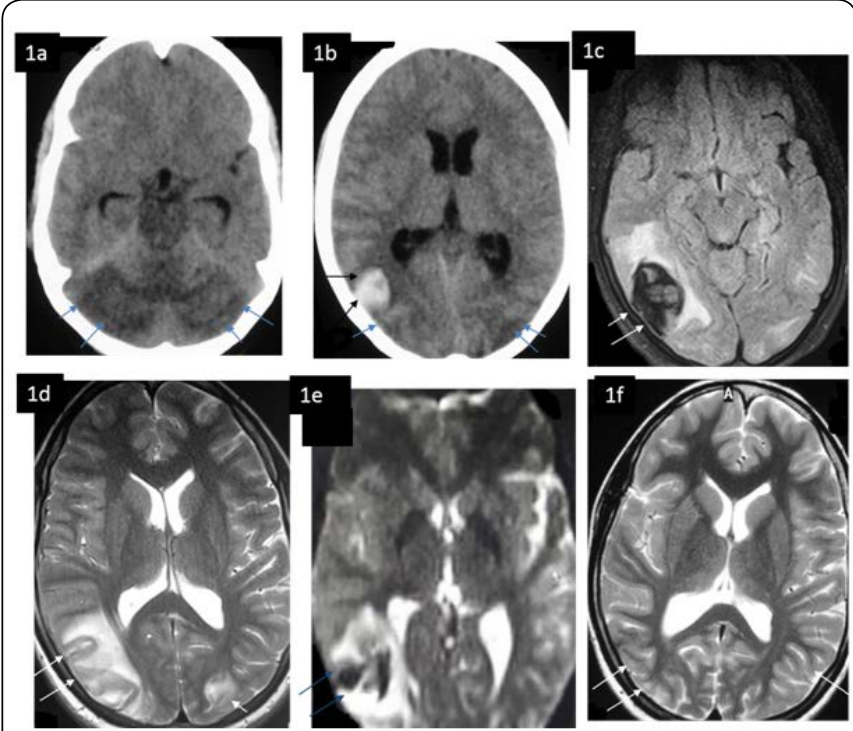

Figure 1: Radiological findings in Posterior reversible encephalopathy syndrome. 1a and 1b: Non-contrast computed tomographic (NCCT) scan of brain showing hypodensities (blue arrows) in brainstem and cerebellar hemispheres on both sides (1a), parietal hemorrhage (1b) and hypodensities (blue arrows) in bilateral parieto-occipital regions (1b). 1c: MR Fluid attenuated inversion recovery sequences of brain showing hypointense signal changes on right side suggestive of hemorrhage (white arrows); 1d: T2 weighted MR sequences showing bilateral temporoparietal hyperintense lesions (right > left) (white arrows); 1e: evidence of diffusion restriction on diffusion weighted imaging (blue arrows); 1 f and 1d: Resolution of signal changes at follow up MR imaging (white arrows). restriction in the right temporo-parietal region (Figure 1e and 1f). MR angiogram and venogram were normal. She improved gradually and was discharged one week after admission. Repeat MRI done after three months showed resolution of signal abnormalities confirming reversibility. However, it did show evidence of old hemorrhage in form of blooming on susceptibility weighted sequences and gliosis on T1 and T2 weighted images. At eight-month follow up, she is doing well with complete recovery.

\section{Discussion}

Pathophysiology of PRES continues to be controversial. Currently two contradictory hypotheses are advocated to explain this rare clinico-radiological syndrome. One of these suggests impaired cerebral autoregulation due to severe hypertension with attendant increased cerebral blood flow as the primary abnormality, whereas the other emphasizes a systemic inflammatory response resulting in cerebral vasoconstriction followed by hypoperfusion, vessel wall damage and vasogenic edema [3].

The diagnosis of PRES rests on characteristic radiological findings in an appropriate clinical setting, especially because clinical symptoms of PRES (headache, vomiting, seizures, altered sensorium and visual abnormalities) are often nonspecific for etiology.

\section{Radiological characteristics of PRES}

With increasing use of MRI in clinical practice, the repertoire of knowledge concerning MRI findings in various neurological disorders is expanding. The same holds true for PRES. While traditionally PRES is represented by bilateral symmetric signal [hyperintensities on $\mathrm{T} 2$ and Fluid attenuation inversion recovery (FLAIR) sequences and hypointense signal changes on T1 weighted MR sequences] involving subcortical white matter in parieto-occipital regions, this pattern was seen in only $26 \%(n=136)$ of patients with PRES in one study [4]. In the same study, four main radiological patterns of PRES were defined. These include (a) Holohemispheric watershed pattern (23\%) with vasogenic edema involving frontal, parietal, occipital and less commonly temporal lobes corresponding to the watershed regions between anterior, middle and posterior cerebral arteries; (b) Superior frontal sulcus pattern (27\%) wherein vasogenic edema involves primarily the frontal lobes along the superior frontal sulci with variable involvement of parietal and occipital lobes; (c) Dominant parietal-occipital pattern (22\%) described conventionally and (d): Partial expression of (a), (b) or (c) (28\%).

In a recent review, [5] of radiological findings in PRES, posterior parietal or occipital lobe $(\geq 98 \%)$ were the most common sites of involvement followed by frontal lobes (70\%), temporal lobes (65\%), cerebellum (30 to 53\%), thalamus (30\%), basal ganglia (11.8-34\%), brainstem (12.5 to $27 \%$ ), and very rarely spinal cord. There is evidence to suggest that while in mild hypertension supratentorial structures are affected dominantly, severe hypertension produces changes primarily in infratentorial structures, basal ganglia and thalamus [5]. 


\section{Hemorrhage in PRES}

Incidence of hemorrhage in PRES ranges from 5\% to $17 \%$ in different case series with almost equal incidence of parenchymal and subarachnoid bleeds [6-8]. The parenchymal bleeds may range in size from small microfoci of hemorrhage to large intraparenchymal hematomas [5]. The reason for hemorrhage is thought to be either rupture of small pial vessels consequent to severe hypertension or reperfusion injury following restoration of cerebral blood flow after vasoconstriction. Hemorrhage in more common in patients with severe edema on FLAIR MR sequences as well as following bone marrow or solid organ transplants [6]. Our patient had a large temporo-parietal bleed, yet she recovered completely. Thus, presence of hemorrhage in PRES is not necessarily associated with poor outcome.

\section{Diffusion restriction in PRES}

Diffusion restriction is reported to occur in $10-23 \%$ of PRES $[4,9]$. It reflects cytotoxic edema and brain ischemia which is thought to result from microcirculatory compromise consequent to severe vasogenic edema. Though earlier, considered to be marker of irreversible brain damage, recent studies have shown that diffusion restriction in PRES is completely reversible $[10,11]$. The complete clinical and radiological recovery in our case also attests to the fact that diffusion restriction in PRES is not necessarily irreversible. Nevertheless, the presence of diffusion restriction should alert physicians to possibility of associated reversible cerebral vasoconstriction syndrome, which should be ruled out by MR or conventional angiogram $[10,11]$.

\section{Contrast enhancement in PRES}

Though conventionally thought to be rare, this finding in seen in about $50 \%$ of cases of PRES and is thought to reflect damage to the blood-brain barrier. Being a common finding, this pattern must be recognized correctly to avoid unnecessary diagnostic errors [4].

\section{Cerebellar involvement in PRES}

In their original description of radiological findings in PRES, Hinchey et al. [1] found cerebellar involvement in only $1 / 15$ patients. However, cerebellar involvement was reported in approximately $1 / 3^{\text {rd }}$ of all cases of PRES in two series [4, 6] published later and in a review [5]. Our patient had severe bilateral cerebellar vasogenic edema on a NCCT scan at first presentation which again stresses the need to keep this atypical presentation in mind to avoid diagnostic errors. Other atypical sites of involvement in PRES include the brainstem (13-18\%) and basal ganglia (7-14\%) [1, 4-6].

To conclude, our patient had many distinct radiological findings that are uncommon in PRES. Despite the presence of hemorrhage and diffusion restriction, she recovered completely, attesting to relatively benign nature of PRES and good prognosis once it is diagnosed and managed appropriately.

\section{Funding Sources}

We did not have any source of funding.

\section{Conflicts of Interest}

We do not have any conflicts of interest.

\section{References}

1. Hinchey J, Chaves C, Appignani B, Breen J, Pao L, et al. 1996. A reversible posterior leukoencephalopathy syndrome. $N$ Engl J Med 34(8): 494-500. https://doi.org/10.1056/NEJM199602223340803

2. Casey SO, Sampaio RC,Michel E, Truwit CL.2000. Posterior reversible encephalopathy syndrome: utility of fluid-attenuated inversion recovery MR imaging in the detection of cortical and subcortical lesions. Am J Neuroradiol 21(7): 1199-1206.

3. Zhang L, Wang Y, Shi L, Cao J, Li Z, et al. 2015. Late postpartum eclampsia complicated with posterior reversible encephalopathy syndrome: a case report and literature review. Quant Imaging Med Surg 5(6): 909-916. https://doi.org/10.3978/j.issn.2223-4292.2015.12.04

4. Bartynski WS, Boardman JF. 2007. Distinct imaging patterns and lesion distribution in posterior reversible encephalopathy syndrome. AJNR Am J Neuroradiol 28(7): 1320-1327. https://doi.org/10.3174/ ajnr.A0549

5. Ollivier M, Bertrand A, Clarencon F, Gerber S, Deltour S, et al. 2017. Neuroimaging features in posterior reversible encephalopathy syndrome: a pictorial review. J Neurol Sci 373: 188-200. https://doi. org/10.1016/j.jns.2016.12.007

6. Lee VH, Wijdicks EF, Manno EM, Rabinstein AA. 2008. Clinical spectrum of reversible posterior leukoencephalopathy syndrome. Arch Neurol 65(2): 205-210. https://doi.org/10.1001/archneurol.2007.46

7. McKinney AM, Short J, Truwit CL, McKinney ZJ, Kozak OS, et al. 2007. Posterior reversible encephalopathy syndrome: incidence of atypical regions of involvement and imaging findings. AJR Am J Roentgenol 189(4): 904-912. https://doi.org/10.2214/AJR.07.2024

8. Hefzy HM, Bartynski WS, Boardman JF, Lacomis D. 2009. Hemorrage in posterior reversible encephalopathy syndrome: imaging and clinical features. AJNR Am J Neuroradiol 30(7): 1371-1379. https://doi. org/10.3174/ajnr.A1588

9. Covarrubias DJ, Luetmer PH, Campeau NG. 2002. Posterior reversible encephalopathy syndrome: prognostic utility of quantitative diffusionweighted MR images. AJNR Am J Neuroradiol 23(6): 1038-1048.

10. Wagih A, Mohsen L, Rayan MM, Hasan MM, Al-Sherif AH. 2015. Posterior reversible encephalopathy syndrome (PRES): restricted diffusion does not necessarily means irreversibility. Pol J Radiol 80: 210216. https://doi.org/10.12659/PJR.893460

11. Ducros A, Boukobza M, Porcher R, Sarov M, Valade D, et al. 2007. The clinical and radiological spectrum of reversible cerebral vasoconstriction syndrome. A prospective series of 67 patients. Brain 130(Pt 12): 30913101. https://doi.org/10.1093/brain/awm256 